\title{
Reliability Assessment and Design of Procedures and Devices in Bariatric Surgery: An Engineering Approach
}

\author{
Fontanella $\mathrm{CG}^{1,2}$, Salmaso $\mathrm{C}^{1}$, Toniolo $\mathrm{I}^{1}$, Polese $\mathrm{L}^{1,3}$, Foletto $\mathrm{M}^{1,3}$ and Carniel EL ${ }^{1,4 *}$ \\ ${ }^{1}$ Centre for Mechanics of Biological Materials, University of Padova, Italy \\ ${ }^{2}$ Department of Biomedical Sciences, University of Padova, Italy \\ ${ }^{3}$ Department of Surgery, Oncology and Gastroenterology, University of Padova, Italy \\ ${ }^{4}$ Department of Industrial Engineering, University of Padova, Italy
}

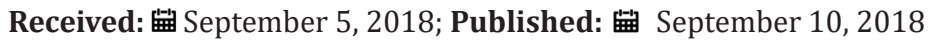

*Corresponding author: Carniel EL, Department of Industrial Engineering, University of Padova, Centre for Mechanics of Biological Materials of Padova, Italy

\begin{abstract}
Bariatric surgery is the most effective intervention for severe obesity, as one of the most serious health problem worldwide. Laparoscopic adjustable gastric banding is one of the principal technique. Nonetheless, side effects are frequent and weight-loss is not always successful. Nonoptimal intervention design, surgery invasiveness and general anesthesia are the principal cause of this situation. A more advanced approach is required, integrating bioengineering and medical competences, aiming to engineering design the procedure, to improve efficacy and to reduce the need for anesthesia. Computational methods can be exploited to evaluate stomach functionality after surgery and to interpret mechano-biological processes, aiming at the optimal design of the intervention. Results from coupled experimental and computational activities are here reported, showing the potentialities of the engineering approach. Endoscopic surgery should minimize invasiveness and anesthetic requirement, but previously proposed techniques demonstrate marginal efficacy. Procedural consistent advances are required, as devices designed to provide endoscopic gastric banding. Preliminary results from computational activities are proposed, again to show the capabilities of the engineering approach to mimic and to optimize the overall surgical procedure.
\end{abstract}

Keywords: Bariatric Surgery; Computational Biomechanics; Endoscopic Surgery

\section{Introduction}

OBESITY is an epidemic disease associated with multiple co-morbidities, whose prevalence in developed countries is increasing. The United States spend million dollars annually on treatment and prevention. It has been estimated that $30 \%$ of adults in the US meet the criteria for obesity and also in Europe obesity is common [1]. Several surveys show that the prevalence of overweight among school age children is as high as $40 \%$ in parts of Europe. The World Health Organization and health agencies in most countries report epidemiological data showing an increase in morbidity and mortality because of increasing weight. Major obesity related diseases include increased risk for cardiovascular diseases, dyslipidemia, diabetes mellitus, cancer, and psychosocial impairment. These conditions are responsible for more than 2.5 million deaths per year worldwide. The loss of life expectancy is profound [2]. Bariatric surgery remains the most effective intervention for patients with body mass index (BMI) of $40 \mathrm{~kg} / \mathrm{m} 2$ or greater or those with BMI of $35 \mathrm{~kg} / \mathrm{m}^{2}$ or greater with specific related co-morbidities [3].

Bariatric surgery envisages different interventions, which are routinely performed by laparoscopy. Some operations reduce the stomach capacity, as gastric banding or gastrectomy, some others reduce the absorbing capacity of gastrointestinal organs, as biopancreatic diversion with duodenal switch; finally, there are operations that exploit both the effects, as gastric by-pass. Nowadays, adjustable gastric banding is one of the most common and successful operation [4]. Surgical failure and post-surgical side effects affect up to $25 \%$ of the patients [3]. Furthermore, the efficacy of bariatric surgery, as post-operative weight loss and approach to food, should be improved. Complications are mostly due to the empirical approach, surgery invasiveness and high anesthesiology risk. For this reason, there is a need for less invasive bariatric procedures, as endoscopic ones. Currently available endoscopic techniques still have limits and mostly fail because of their unsuitableness or procedural difficulties [5]. Further efforts are mandatory for a reliable definition of endoscopic methods. Coupled engineering and clinical activities are necessary for the design, as an example, of a reliable and effective endoscopic approach to gastric banding.

The specific bariatric procedure and the post-surgical conformation of the operated gastrointestinal organs are usually 
defined on clinical basis only. A more rational approach is advocated, considering physiological and mechano-biological processes, as the physio-mechanical functionality of the gastrointestinal organs and the relationship between food intake, mechanical stimulation of gastrointestinal wall and feeling of satiety. Methods and procedures of bariatric surgery should be consequently defined by integrating competences in the areas of surgery, physiology and bioengineering. In this context, only qualitative or semi-quantitative activities have been previously performed [6,7]. Considering the specific bariatric procedure, mechanical models of the gastrointestinal tract can be developed and applied to quantitatively investigate the mechanical stimulation of the gastrointestinal wall because of food intake [8]. Physiological and mechano-biological investigations can be performed to identify quantitative relationships between mechanical stimulation of the gastrointestinal wall, volume of the ingested food and feeling of satiety. The methods of computational biomechanics, mechano-biology, physiology and surgery can be exploited aiming at defining computational tools for the optimal design of procedures and devices for a more reliable and effective approach to bariatric surgery.

\section{Materials and Methods \\ Computational Model of the Stomach}

Aiming at providing computational tools for bariatric surgery optimization, a computational model of the stomach was developed. Model definition and validation were performed by analyzing histo-morphometric data and results from mechanical tests at both tissue and structure levels. An inner connective layer and an outer muscular layer mainly compose the stomach wall. Collagen and muscular fibers within connective and muscular layers, respectively, show preferential orientations, leading to anisotropic behavior. Thickness measurements, histological investigations [9] and mechanical tests on wall samples [10] from the different regions of the stomach (fundus, corpus and antrum) pointed out different results. Consequently, the stomach wall can be defined as a multi-layered, anisotropic and non-homogeneous structure (Figure 1a). Aiming at characterizing stomach tissues mechanics, results from experimental investigations suggested the assumption of a visco-hyperelastic constitutive formulation:

$$
S\left(C_{2} q^{i}\right)=2 \frac{\partial W^{0}(C)}{\partial C}-\sum_{i=1}^{n} q^{i}
$$

where $\mathrm{S}$ is the second Piola-Kirchhoff stress tensor, $\mathrm{C}$ is the right Cauchy-Green strain tensor, while is an hyperelastic potential that specifies the instantaneous response of the tissue and was defined considering the typical fiber-reinforced configuration [11]. The evolution of viscous variables was specified by usual differential equations:

$$
q^{i}+\frac{1}{\tau^{i}} q^{i}=2 \frac{\gamma^{i}}{\tau^{i}} \frac{\partial W^{0}(C)}{\partial C}
$$

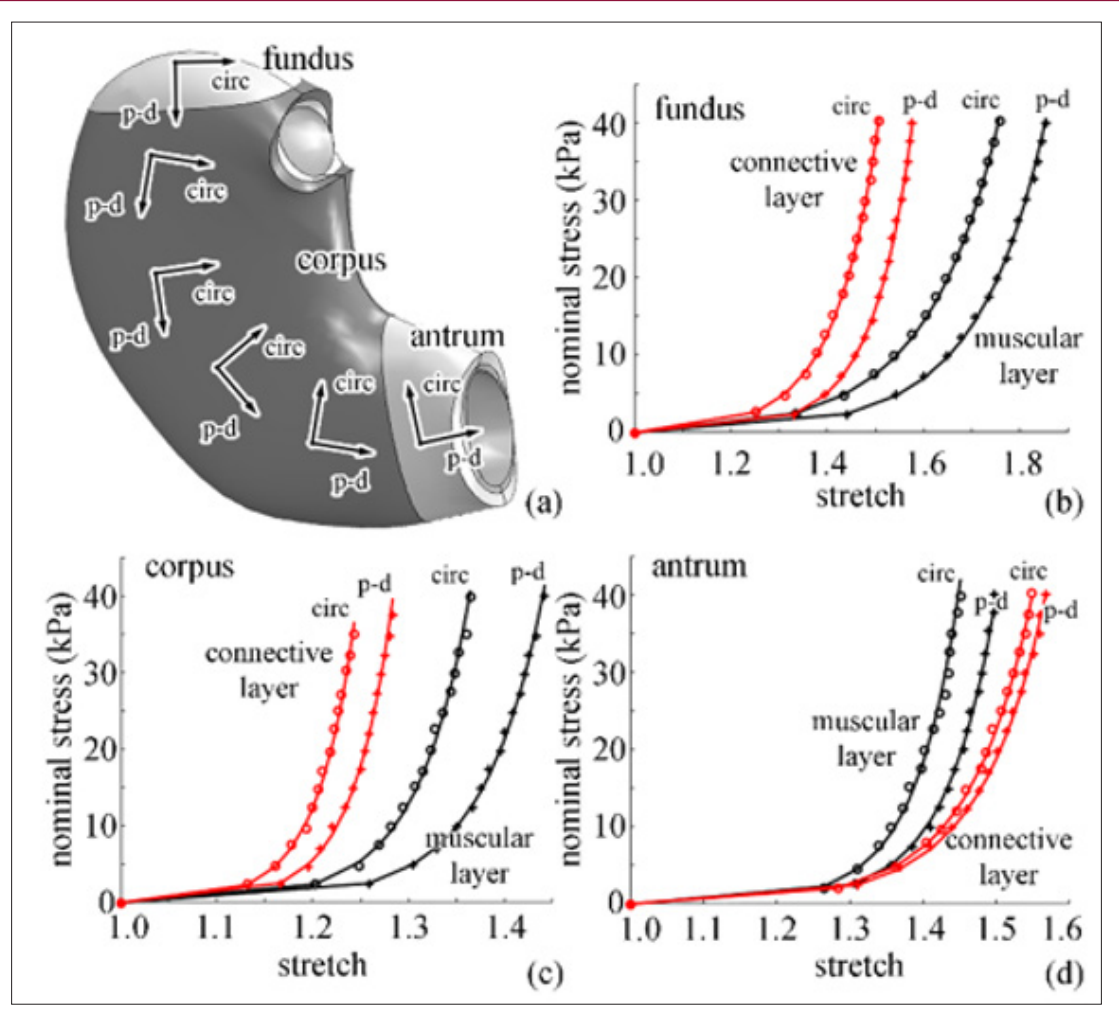

Figure 1: Virtual solid model of the stomach with indication of the three main regions, as fundus, corpus and antrum, and the principal orientations, as proximal-distal and circumferential directions (a). Inverse analysis of tensile tests on swine stomach tissues specimens from different regions, as fundus (b), corpus (c) and antrum (d), and layers: experimental data (empty circles, as results from tests performed along circumferential direction, and crosses, as results from tests performed along proximal-distal direction) and model results (continuous lines). 
Hyperelastic parameters have been identified by the inverse analysis of uni-axial tensile tests (Figure $1 \mathrm{~b}$-d) which have been performed on wall samples from pig stomachs [10]. In detail, specimens from connective and muscular layers were harvested from the fundus, the corpus and the antrum, considering both proximal-distal and circumferential directions (Figure 1a). On the other side, viscous parameters were identified by the analysis of relaxation data from structural tests, as reported below. Aiming at the definition and the validation of the stomach computational model, structural tests were performed considering stomach samples [8]. Stomachs of fifteen piglets (weight: $30 \pm 2 \mathrm{Kg}$ ) were collected. Detailed stomach measurements were performed. Subsequently inflation tests allowed to evaluate the stomach structural response, as the pressure-volume behavior. Sample experimenting was performed by a multi-step inflation procedure. Each step was composed of $280 \mathrm{ml}$ inflation of saline at $40 \mathrm{ml} / \mathrm{s}$ inflation rate, and subsequent $600 \mathrm{~s}$ of rest to allow the almost complete development of relaxation phenomena. The step was repeated up to the full filling of the stomach, leading to pressuretime data.

The collection of pressure and volume data at the end of the rest stages led to pressure-volume almost equilibrium curves. The analysis of pressure-time results during the constant volume stages led to relaxation curves, entailing the identification of the viscous parameters. Experimentations were performed considering both the pre- and the post-bariatric surgery conformations. Gastric banding was provided by a silicon rubber band, evaluating also the influence of band pre-tension. Morphometric data were processed to develop an average finite element model of the fundus, the corpus and the antrum, considering both the connective and the muscular layers. A distribution of local coordinate systems was defined to provide a mathematical description of the orientation of collagen and muscular fibers (Figure 1a). The mechanical behavior of the stomach tissues was defined by implementing the anisotropic visco-hyperelastic formulation in the framework of the general purpose finite element code Abaqus Explicit 6.14 (Dassault Systèmes Simulia Corp., Providence, RI). Computational analyses were performed aiming to investigate the pressure volume behavior of the stomach. Subsequently, the stomach computational model was morphed according to data from measurements performed on human samples [9].

\section{Investigation of Stomach Functionality in Post-Surgical Conformation}

The post-surgical conformation of the stomach is usually defined on the basis of the surgeon expertise and the outcome of previously performed interventions. Activities are required to prove methods for a rational pre-surgical planning of the bariatric intervention. Computational analyses can be performed to evaluate stomach functionality in the post-surgical conformation, as the pressure-volume behavior and the mechanical stimulation of gastric receptors. As an example, the computational model of the stomach was exploited to investigate the stomach mechanical functionality after laparoscopic gastric banding, depending on the band pre-tension condition (Figure 2). A finite element model of the silicon rubber band was developed. Band pre-tension was simulated considering a fictitious thermal contraction of the band.

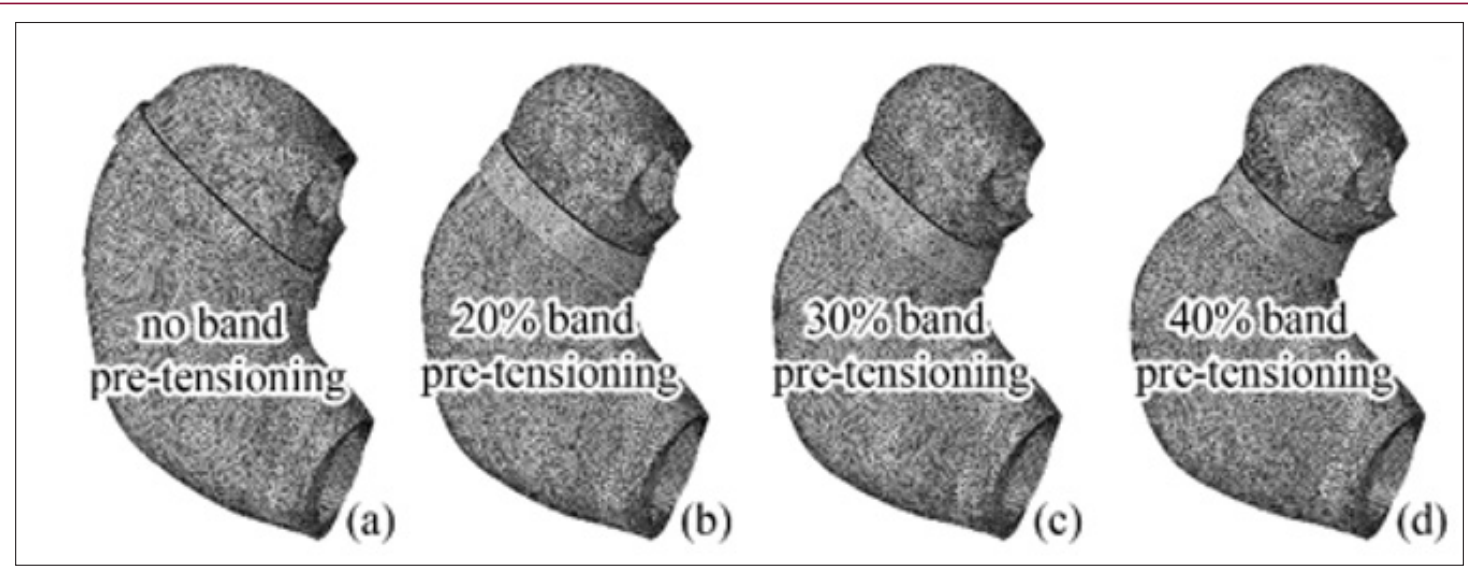

Figure 2: Computational modeling of laparoscopic gastric banding. Models are developed considering also the influence of band pre-tensioning, as $0 \%$ (a), $20 \%$ (b), 30\% (c) and $40 \%$ (d) band stretch. Models are exploited to investigate tissues response because of stomach inflation. A fluid filled cavity is defined considering the stomach inner surface and a constant fluid incoming flow is imposed. The upper and the lower extremity of the cavity, at the gastroesophageal and gastroduodenal junctions, are fixed.

\section{Tools for Endoscopic Approach to Gastric Banding}

Bariatric surgery is mainly developed by laparoscopic approach, which requires general anesthesia. The anesthesiology risk in obese patient is relevant. Endoscopic techniques are less invasive and sedation requirement is minor, but efforts are mandatory to define a reliable approach. Computational methods can be exploited for both the evaluation of stomach functionality in post-surgical conformation and the design of devices and instrumentations for the endoscopic methods. As an example, an endoscopic approach to gastric banding was analyzed. The intervention should be performed affixing a circular pattern of clips in the stomach fundus all around the gastroesophageal junction. Subsequently, clips must be connected by a wire (Figure 3). 


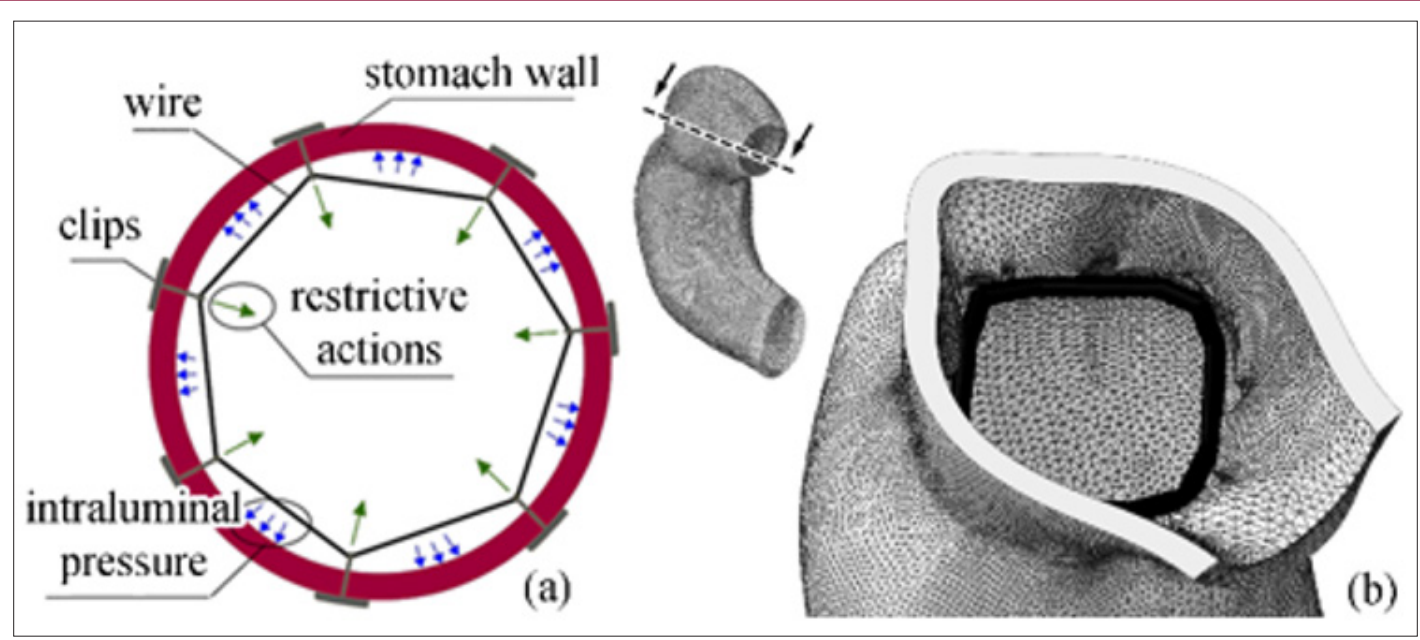

Figure 3: Schematic representation (a) and computational investigation (b) of the endoscopic approach to gastric banding.

Computational analyses have been performed to identify a reliable design of the clips. Wire traction allows the development of the stomach pouch, which is typical of gastric banding. The major efforts pertain to design reliable surgical clips, considering both the applicability by an endoscopic tool and the capability to support wire traction. Different clips conformations have been investigated, as anchors, spiders, screws and spirals (Figure 4).

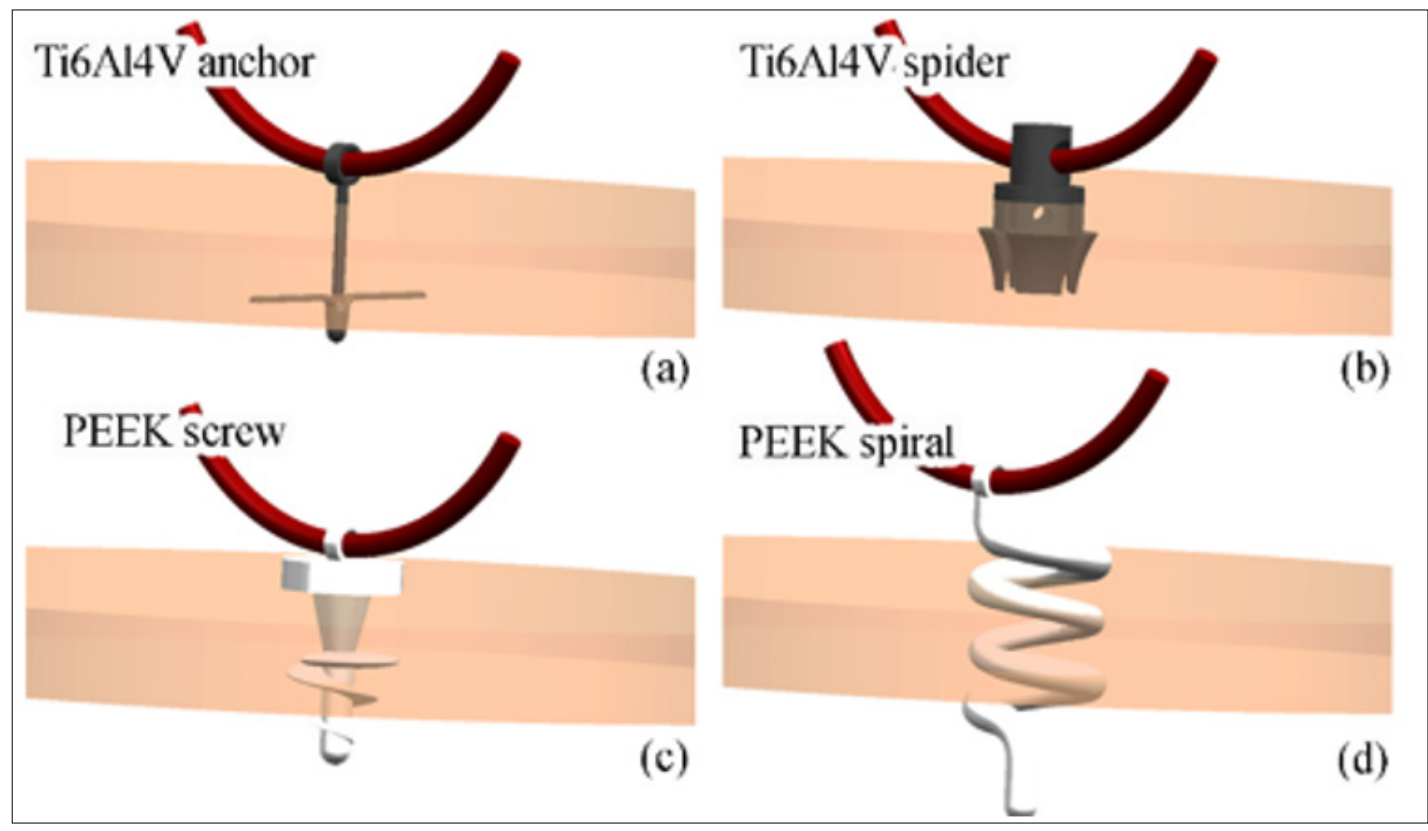

Figure 4: Virtual solid models of different clips for endoscopic gastric banding:
a. Anchor,
b. Spider,
c. Screw and
d. Spiral.

\section{Results}

The reliability of the computational model of the stomach was assessed by the comparison of experimental and model results from inflation tests (Figure 5). Results are provided considering different stomach conformations, as pre-surgical one and post-surgical conditions after laparoscopic gastric banding. Subsequently, after the morphing procedure in the light of human stomach dimensions, the computational model was exploited to evaluate the influence of gastric banding parameters on stomach functionality, as the pressure-volume behavior and the mechanical loading of gastric wall, with particular regard to the fundus region where mechanoreceptors are mainly localized (Figure 6). Again, reference is made to laparoscopic gastric banding. With regard to the design of the endoscopic approach, preliminary results from the computational analysis of clips insertion are finally proposed (Figure 7). 


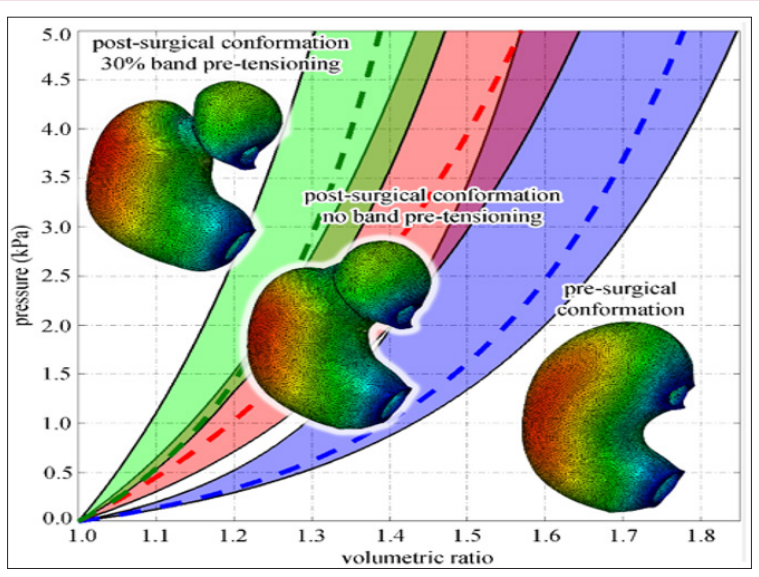

Figure 5: Reliability assessment of the stomach computational model by the comparison of experimental (colored bands) and computational (discontinuous lines) results from inflation tests: pre-surgical conformation (blue), post-surgical conformation with (green) and without (red) band pre-tensioning. Contours of displacement magnitude fields are reported for the different situations (1000 $\mathrm{ml}$ inflated volume).

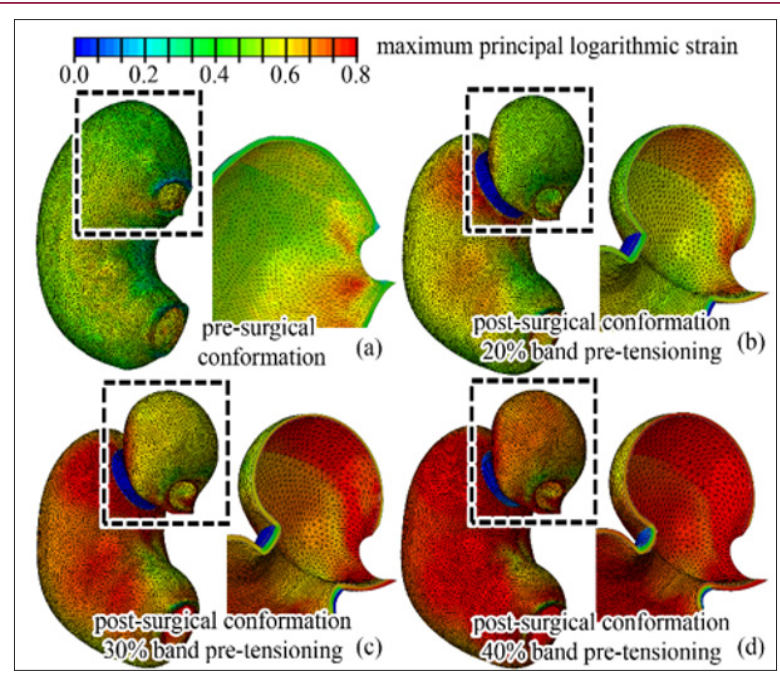

Figure 6: Analysis of stomach functionality in pre- (a) and post- (b-d) surgical conformations depending on gastric band pretensioning. Distribution of tensile strain on the outer surface of the overall stomach and the inner surface of the fundus region. Results are reported at $1000 \mathrm{ml}$ inflated volume.
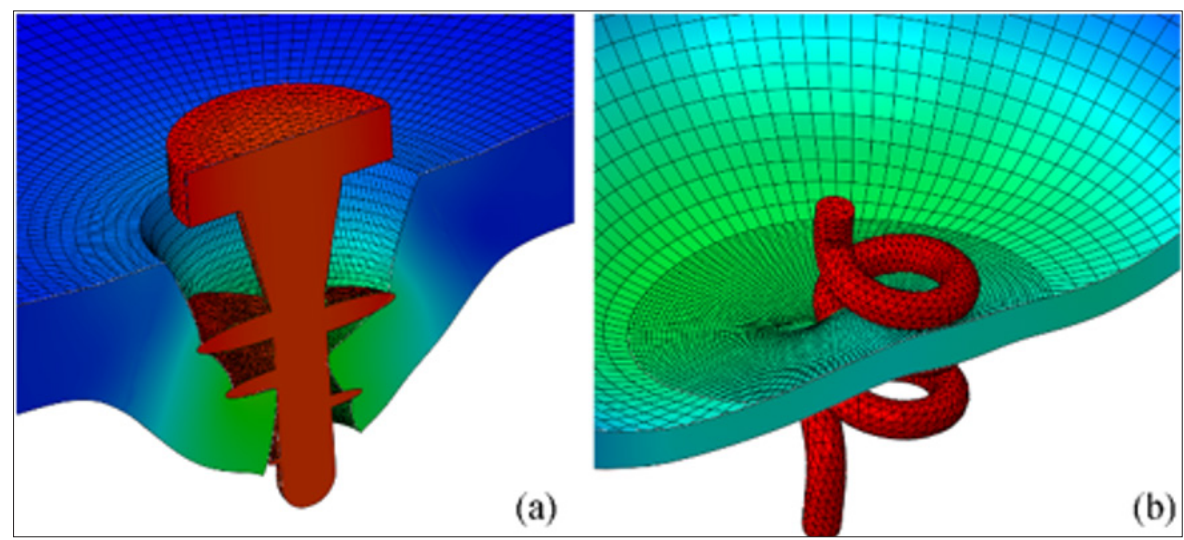

Figure 7: Computational methods for the design of clips for endoscopic wire fixation: analysis of interaction phenomena between clips, as

a. screw and

b. spiral and stomach wall during clip insertion. 


\section{Discussion}

Preliminary outcomes were reported from activities that are under development in the research field of stomach mechanics and bariatric surgery. Results from experimental tests were exploited to develop, identify and validate a computational model of the stomach. Computational activities analyzed stomach natural configuration and different post-surgical conformations. Computational results highlighted the great influence of bariatric surgery parameters on stomach mechanics. In detail, the surgical procedure strongly affects the stomach structural stiffness (Figure 5) and the strain field conformation and intensity (Figure 6). Attention was here focused on gastric banding, because of its wide application and effectiveness. Nevertheless, the same approach can be adopted to analyze other surgical techniques and to design innovative and more reliable procedures (Figures 3,4,7). The results show the potentiality of experimental and computational biomechanics for the investigation of stomach functionality and the planning of bariatric surgery procedures and techniques.

\section{Conclusion}

As previously reported by other authors [7], experimental activities allow evaluating stomach pressure-volume behavior depending on surgical methods and conditions. The increased stomach stiffness after surgery confirms the clinical evidence that bariatric intervention reduces the stomach capacity. On the other side, computational methods make it possible to broad experimental results to an extremely wider scenario, considering many different surgical procedures. Furthermore, computational models provide information that experimental methods cannot supply. In detail, the computational approach makes it possible to identify the strain and the stress fields in stomach tissues, whose distribution is strongly affected by the bariatric procedure. As an example, position and pre-tension value of the gastric band have a relevant impact on both the stomach capacity (Figure 5) and the areas of gastric wall that are mainly strained and stressed (Figure 6). Mechanical stimuli, as strain and stress, act on receptors that contribute to regulate the feeling of satiety. The relevance of this aspect is enforced by the recent trends in bariatric surgery, which aim not to restrict stomach capacity, but to induce satiety by increasing receptors stimulation [6].

Considering the mechano-transduction capabilities of gastric receptors, it follows the relevance of the proposed computational methods, which allow evaluating the intensity and the conformation of strain and stress fields depending on stomach conformation and food intake. Further efforts are mandatory for a more exhaustive and accurate computational approach to stomach mechanics. For example, the here reported experimental activities have been developed considering biological samples from pig model only. Activities are currently under development adopting human samples that are harvested from stomach scraps from bariatric interventions. Furthermore, a more reliable investigation of stomach mechanics should consider motility, as the active contribution of muscular components, and fluid-structure interaction phenomena between bolus and stomach wall. Such approach should entail a more reliable investigation of physio-mechanical processes that develop during stomach activities. In conclusion, computational methods enable the evaluation of bariatric intervention reliability and effectiveness, and the functional comparison between different surgical techniques. Computational tools can consequently provide a valuable aid for the pre-surgical design and the definition of novel procedures.

\section{References}

1. Gallus S, Lugo A, Murisic B, Bosetti C, Boffetta P, et al. (2015) Overweight and obesity in 16 European countries. Eur J Nutr 54(5): 679-689.

2. Berenson GS (2012) Health Consequences of Obesity. Pediatr Blood Cancer 58: 117-121.

3. Chang SH, Stoll CR, Song J, Varela JE, Eagon CJ, et al. (2014) The effectiveness and risks of bariatric surgery: an updated systematic review and meta-analysis, 2003-2012. JAMA Surg 149(3): 275-287.

4. Lazzati A, De Antonio M, Paolino L, Martini D, Azoulay A, et al. (2017) Natural history of adjustable gastric banding: lifespan and revisional rate: a nationwide study on administrative data on 53,000 patients. Ann Surg 265(3): 439-445.

5. Choi HS, Chun HJ (2017) Recent Trends in Endoscopic Bariatric Therapies. Clin Endosc 50(1): 11-16.

6. Woods SC (2004) Gastrointestinal satiety signals I. An overview of gastrointestinal signals that influence food intake. Am J Physiol Gastrointest Liver Physiol 286(1): 7-13.

7. Gao F, Liao D, Zhao J, Drewes AM, Gregersen H (2008) Numerical analysis of pouch filling and emptying after laparoscopic gastric banding surgery. Obes Surg 18(3): 243-250.

8. Carniel EL, Frigo A, Fontanella CG, De Benedictis GM, Rubini A, et al. (2017) A biomechanical approach to the analysis of methods and procedures of bariatric surgery. J Biomech 56: 32-41.

9. Elariny H, González H, Wang B (2005) Tissue thickness of human stomach measured on excised gastric specimens from obese patients. Surg Technol Int 14: 119-124.

10. Zhao J, Liao D, Chen P, Kunwald P, Gregersen H (2008) Stomach stress and strain depend on location, direction and the layered structure. J Biomech 41(16): 3441-3447.

11. Carniel EL, Gramigna V, Fontanella CG, Stefanini C, Natali AN (2014) Constitutive formulations for the mechanical investigation of colonic tissues. J Biomed Mater Res A 102(5): 1243-1254. 
ISSN: 2574-1241

DOI: $10.26717 / B J S T R .2018 .08 .001717$

Carniel EL. Biomed J Sci \& Tech Res

(c) (P) This work is licensed under Creative BY Commons Attribution 4.0 License

Submission Link: https://biomedres.us/submit-manuscript.php

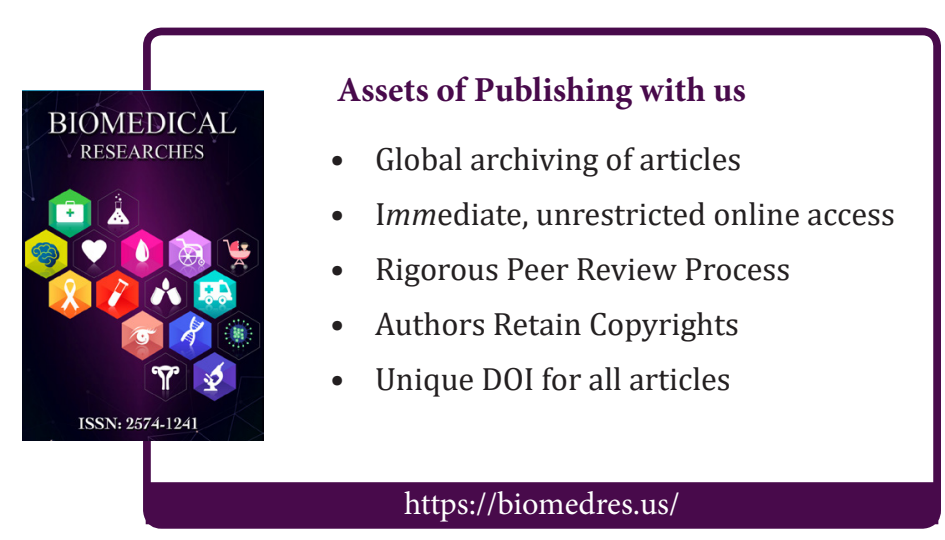

